\title{
Multilinguales
}

\section{Pour une analyse des savoirs enseignés en classe de langue}

For an Analysis of the Knowledge Taught in a Language Class

\section{Khedoudja Meziane et Abdenour Arezki}

\section{(2) OpenEdition \\ 1 Journals}

Édition électronique

URL : http://journals.openedition.org/multilinguales/912

DOI : $10.4000 /$ multilinguales. 912

ISSN : 2335-1853

Éditeur

Université Abderrahmane Mira - Bejaia

\section{Édition imprimée}

Date de publication : 1 juin 2015

Pagination : 03-26

ISSN : 2335-1535

\section{Référence électronique}

Khedoudja Meziane et Abdenour Arezki, « Pour une analyse des savoirs enseignés en classe de langue », Multilinguales [En ligne], 5 | 2015, mis en ligne le 01 juin 2015, consulté le 17 septembre 2019. URL : http://journals.openedition.org/multilinguales/912; DOI : 10.4000/multilinguales.912

Ce document a été généré automatiquement le 17 septembre 2019.

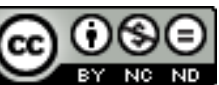

Multilinguales est mise à disposition selon les termes de la Licence Creative Commons Attribution Pas d'Utilisation Commerciale - Pas de Modification 4.0 International 


\section{Pour une analyse des savoirs enseignés en classe de langue}

For an Analysis of the Knowledge Taught in a Language Class

Khedoudja Meziane et Abdenour Arezki

L'intégration du concept de transposition didactique à l'appareillage conceptuel de la didactique ainsi que le développement du champ de l'analyse du discours en sciences du langage ont joué en faveur de l'émergence de nouvelles perspectives de recherche, à l'exemple de l'analyse du discours pédagogique. Celui-ci fait actuellement l'objet de plusieurs travaux qui portent, entre autres, sur l'étude des objets enseignés à travers les phénomènes de didacticité ${ }^{1}$, dont en particulier la reformulation (C. Garcia-Debanc, 2006), et sur les stratégies de guidage (M. Waendenries, 1996). Ces recherches s'inscrivent dans ce qui est appelé par J-F. Halté (2005), la "version forte» des interactions didactiques, c'est-à-dire celle qui considère que ces dernières constituent l'« activité même de l'enseignement " (Ibidem). Nous avons donc affaire à un outil de coconstruction du savoir et non pas à un simple canal par le biais duquel cette coconstruction est réalisée: organisation des tours de parole, sollicitation des apprenants, etc.

1 C'est dans cette perspective que s'inscrit la présente étude qui a pour objet l'analyse d'une variable du discours didactique: les savoirs enseignés en tant qu'objets transposés appartenant à différents champs disciplinaires. Il s'agit de rendre compte de la nature de ces objets et de la manière choisie pour leur transposition, et ce, en vue d'apprécier leur potentiel à favoriser la construction, chez l'apprenant, d'une représentation cohérente de l'écrit.

\section{Savoirs enseignés, séquences et noyaux de didactisation}

2 Dans cette étude, nous partons du postulat que certains savoirs sur la langue et autres sont plus propices à cette construction. C'est le cas, à titre d'illustration, de notions 
actuelles en didactique qui constituent des données théoriques largement admises dans cette discipline et dans d'autres auxquelles elle fait référence, à savoir les sciences du langage et la psychologie cognitive. Il s'agit donc de données constituant le fruit de dizaines d'années de recherche, caractérisées par de nombreux remaniements: supplantation de la notion de type par celle de genre, intégration de la dimension textuelle dans l'enseignement, adoption des théories constructivistes et socioconstructivistes dans l'élaboration pédagogique, etc.

3 Les savoirs enseignés ne sont pas les seuls à nous intéresser dans le présent article, mais aussi la manière dont ils se présentent à travers le corpus analysé, leur progression. Cet aspect est également révélateur de l'existence d'un potentiel de nature à favoriser ou non la mise en place d'une compréhension efficace de l'écrit. Afin de rendre compte de toutes ces dimensions d'analyse, nous avons conçu les notions de séquence et de noyau de didactisation. Nous entendons par la première un faisceau de savoirs articulé autour d'un objet principal donné et entretenant avec d'autres un certain rapport. Nous appelons chacun des savoirs en question noyau de didactisation, qui, dans le cadre concret d'une séance pédagogique, correspond à une notion ou un concept que l'enseignant s'assigne à faire comprendre à l'apprenant à travers les échanges le liant à celui-ci.

4 Les noyaux de didactisation constituent les savoirs effectivement enseignés, lesquels résultent d'un processus de sélection et de transformation établi sur les savoirs enseignables, c'est-à-dire les objets théoriques préalablement sélectionnés et simplifiés lors d'une transposition didactique préliminaire. Notons que l'apprenant peut être à l'origine de certains noyaux de didactisation, mais ceux-ci ne sont considérés comme tels que lorsqu'ils sont validés par l'enseignant. Seule cette validation leur confère le statut d'objets didactisés car la didactisation dont il s'agit dans notre recherche est du ressort de l'enseignant.

5 Par ailleurs, il est à souligner que nous distinguons une catégorie particulière de noyaux de didactisation que nous avons qualifiés d'indépendants. Ces derniers ne figurent pas dans le cadre des séquences de didactisation, dont chacune comporte au moins deux noyaux, mais en dehors de celles-ci. Ils se distinguent, par ailleurs, par le fait qu'ils peuvent ne pas relever initialement du projet explicatif de l'enseignant, mais survenir à l'occasion d'un événement marginal produit en classe (Cf. infra. Les noyaux $B$ et C).

6 Enfin, nous précisons que notre étude a pour corpus d'analyse le discours enregistré et transcrit d'un enseignant expérimenté, recueilli à l'université de Bejaia, en fin de l'année 2011. Le discours en question est produit en situation d'interaction dans le cadre de deux séances d'enseignement de la matière "Ecrit». Au cours de ces dernières, s'étalant sur une durée globale d'environ 5 h, l'objet « Ecrit », en particulier dans sa dimension textuelle, est abordé. Si notre analyse prend sens en dehors de toute considération liée à une quelconque répartition dans le temps (volume horaire/ organisation en séances), nous avons jugé néanmoins utile, pour une meilleure lisibilité, de la répartir suivant les deux séances pédagogiques au sein desquelles se situe le discours d'enseignement étudié. 


\section{Les séquences et les noyaux de didactisation dans la séance 1}

Dans la première séance d'enseignement analysée, nous distinguons 11 séquences de didactisation parmi lesquelles nous comptons 4 principales, 5 dérivées et 2 enchâssées. Nous relevons également 3 noyaux de didactisation indépendants dont un est dérivé. Deux moments principaux marquent le discours de l'enseignant dans la séance analysée. Le premier s'articule sur l'écrit, ses étapes (séquences 1 et $1^{\circ}$ ) et ses conditions de production (séquences $2,2^{\circ} \mathrm{a}, 2^{\circ} \mathrm{b}$ ) ; le deuxième est construit autour de l'objet « texte » (noyau A), sa cohérence (séquence 3), ses variantes (séquence 4) et les deux métarègles de cohérence que sont la progression (noyau $3^{\circ} \mathrm{a}$, séquence $3^{\circ} \mathrm{aa}$ ) et la répétition (séquence $3^{\circ} \mathrm{b}$ ) ${ }^{2}$.

Séquence de didactisation 1 : autour des étapes permettant la production de l'écrit

L'écrit est étudié en situation de compréhension et en situation de production ..........comme pour l'oral...........Pour produire, il faut se mettre à écrire..........pour arriver à se mettre à écrire il faut comprendre..........comprendre la manière dont on a écrit......... il y a un certain nombre de fonctionnements limités de textes ce qui permet d'apprendre à les écrire......... après avoir compris le fonctionnement d'un texte, on doit, pour pouvoir l'écrire, le reproduire.......... écrire tel type de texte.........texte narratif, texte expositif, texte argumentatif, texte explicatif, texte descriptif......... on peut à la rigueur, après, prendre la liberté qu'on veut pour pouvoir produire n'importe quel texte [...] à notre manière.

Dans cette séquence, le rapport entre l'activité de compréhension et celle de production est mis en évidence: la production, proposée comme un but principal, nécessite toute une série de phases rattachées directement à la compréhension. Cette dernière implique de s'intéresser aux différents éléments qui composent un texte et le structure ; autrement dit, les éléments de son fonctionnement qui le situent dans la sphère d'un type particulier, le distinguant d'autres textes. Arrivé à la phase d'écriture, le scripteur rédigera suivant les caractéristiques du type en question. Ce n'est qu'après que le sujet écrivant pourrait donner à son écrit une empreinte personnelle caractéristique de son propre style. Cheminement logique dans son ensemble qui rentre dans la conception communément admise de la dépendance de l'acte de produire à celui de comprendre comme le soutiennent ceux qui prônent l'enseignement/apprentissage articulé des compétences, dont nous citons S. Moirand (1979 : 98), J-P. Cuq \& I. Gruca (2003 : 188) et M. Ammouden (2012).

7 Cependant, ce qui est, présente un fait, aujourd'hui largement contesté aussi bien sur la scène linguistique que didactique, dans le contenu de la séquence précédente, c'est l'adoption de la notion de typologie. Notons que J-M. Adam (2005), qui était l'un des principaux théoriciens des types de textes, explique, dans son article, intitulé «La notion de typologie de textes en didactique du français : une notion «dépassée »? ", pourquoi la notion de typologie s'avère finalement non rentable sur le plan didactique. En effet, moins pertinente, cette notion prise au sens entendu par l'enseignant est délaissée au profit de celle de genre. Le genre, par son aspect d'objet complexe, et par son caractère social qui le situe dans l'aire sociale de la production langagière, est celui actuellement mis en valeur par les théoriciens de l'approche actionnelle, véhiculée par le Cadre Européen Commun de Référence pour les Langues (Conseil de l'Europe, 2001). Dans un ouvrage traitant de la Progression dans l'enseignement du français langue première, S-G. Chartrand et al. (2008: 19) se réfèrent à plusieurs auteurs en faveur de cette nouvelle orientation supplantant les anciennes typologies de textes : 
L'entrée dans l'univers foisonnant de la communication langagière par l'étude de plusieurs textes d'un même genre fournit à l'élève de puissants outils pour la compréhension et la production de ce même genre, comme le montrent plusieurs recherches empiriques et didactiques: Canvat, 1994; David-Chevalier, 2005; De Pietro et Schneuwly, 2003; Dolz et Schneuwly, 1998 ; Privat-Vinson, 1988 ; Reuter, 2007 ; Schneuwly, 2002, 2007 b.

Il faut souligner qu'en dehors du choix non pertinent de la notion de typologie textuelle, le principe lui-même, celui de distinguer l'individuel du social, le particulier du conventionnel, en parlant des notions de type de texte et de style personnel dans l'approche de l'écriture, est à percevoir positivement du point de vue de l'apprentissage. En effet, mettre en lumière cette réalité, sans doute non méconnue, du moins intuitivement, de l'apprenant auquel s'adresse l'enseignant, est susceptible d'entraîner à une prise de conscience des plus précieuses pour l'appropriation de la langue. ${ }^{3}$

8 Par ailleurs, un noyau de la séquence de didactisation précédente (Cf. supra Séquence de didactisation1), la compréhension, forme à son tour une nouvelle dérivée :

Séquence de didactisation $1^{\circ}$ : autour de la notion de « comprendre un texte "

Comprendre un texte c'est comprendre sa signification...........'est retrouver ses

idées.........(séquence enchâssée) Dans un texte il y a des idées......... et la forme

.........que prennent ces idées.........(retour à la séquence $1^{\circ}$ ) on peut dire que j'ai compris le texte au moment où on comprend comment fonctionne les idées dans leur forme..........(retour à la séquence enchâssée) un texte est un tout y compris son image.........(.retour à la séquence $1^{\circ}$ ) ..........comprendre un texte est comprendre ce qui est dit là [...] ça ne veut absolument rien dire parce qu'il se peut qu'il ait quelque chose qui puisse nous échapper.

Le noyau compréhension est repris ici dans le but d'amener à la correction d'une représentation courante de l'activité qu'il désigne chez les apprenants ; représentation selon laquelle la compréhension implique une simple identification des idées du texte. L'enseignant explique que cette activité cognitive qui est la compréhension n'est pas aussi simple qu'on la croit car elle implique de comprendre les idées à travers leur mise en texte. Mais, les propos de l'enseignant se voient contredits en fin de la séquence et l'équilibre de cette dernière subit une rupture totale. En effet, l'enseignant avance que la compréhension d'un texte ne signifie nullement comprendre son sens car il y aurait quelque chose d'inéluctable qui échapperait au lecteur.

L'absence de toute allusion au rôle prépondérant des acquis dans la présentation du phénomène de compréhension ci-dessus est une autre insuffisance de la séquence précédente. C'est ignorer un consensus établi en psychologie cognitive sur le fait que le sujet mobilise tout un dispositif de connaissances stockées dans sa mémoire à long terme pour en construire de nouvelles (Piaget cité par M. Wambach \& Ciaver, 2001).

Par ailleurs, sur le plan structurel, une séquence de didactisation est enchâssée dans la précédente. Elle est reconnaissable par sa formulation autour du thème de texte. Cette séquence, qui apparaît à deux moments différents, traite de la définition du texte. Néanmoins, elle n'est pas à percevoir comme intruse car elle travaille clairement l'objectif principal, la définition de la compréhension d'un texte: il faut définir le « texte " pour savoir ce qu'est " comprendre un texte ».

Dans la continuité de la séquence de didactisation 1 , s'inscrit la séquence 2 , qui se présente de la manière suivante :

Séquence de didactisation 2 : autour des conditions de production de l'écrit

Pour qu'il y ait un écrit, il faut des conditions: stylo, feuille..........il faut

obligatoirement une personne qui va écrire..........et une intention..........qui une fois 
" graphé » permet de partager l'écrit avec le lecteur..........dans le texte, il y a un message.........matérialisé dans un code écrit.........par le biais d'un canal.........et il y a un référent.

Ainsi, les deux séquences de didactisation 1 et 2 (cf. supra), répondent à la question principale, « de quoi faudra-t-il disposer pour écrire?». Si la première apporte trois principaux éléments de réponse, à savoir comprendre le fonctionnement du texte, le reproduire dans sa forme conventionnelle, puis prendre la liberté possible dans l'écriture, la deuxième énumère les différentes conditions de cette reproduction/ production de l'écrit au moment de l'accomplissement de l'acte d'écriture, lors de la mise en œuvre de la compréhension dont il est question dans la séquence1. Il s'agit là des conditions matérielles (stylo, feuille) et des six éléments du schéma de la communication de R. Jakobson (1963 : 213-214).

11 De prime abord, nous pouvons signaler que la conception de la communication empruntée à Jakobson a fait l'objet de plusieurs remises en cause. Certes le modèle constitue lui-même un enrichissement d'autres dits "techniques» qui l'ont précédé, dans la mesure où il introduit la notion de contexte avec ses deux composantes: l'environnement linguistique des unités de sens et les conditions sociales où se déroule la communication (D. Picard, 1992: 73). Cependant, il ne va pas jusqu'au bout de l'entreprise et se limite à évoquer par exemple les conditions sociales, telles que l'appartenance culturelle des communicants, "sans réellement leur donner un statut précis » (Ibidem : 74-75).

12 L'ordre des noyaux de didactisation de la séquence 2 est aussi discutable. La personne avec son intention de communication ne constitue-t-elle pas en effet la condition première de l'écriture ? Toutefois, l'avantage que revêt la structuration observée réside dans la façon graduelle dont sont abordées les notions. L'enseignant commence d'abord par évoquer les éléments matériels, les plus concrets, mais aussi les plus évidents, le stylo et la feuille, avant de passer à d'autres, à savoir les éléments du schéma de la communication de Jakobson. Nous pensons que ce genre de techniques amènerait l'apprenant à se forger une idée cohérente des conditions de production de l'écrit dans la mesure où elle permettrait d'atténuer les contraintes de l'acte de répondre. L'apprenant pourrait induire de la technique précitée le sens suivant : «Il n'y a rien de difficile dans la question posée, tout est abordable pourvu que l'on procède de façon simple et progressive, sans rechercher de complication ». Ceci nous rappelle le vieux principe didactique de la méthode directe, aller du concret vers l'abstrait (C. Germain, 1993, C. Puren, 1988), sauf qu'ici il est appliqué à l'enseignement des faits «sur la langue » et non « de la langue ».

13 Le message et le référent seront repris dans d'autres séquences de didactisation. Dans la séquence $2^{\circ}$ a (Cf. infra), ces deux notions, pouvant présenter d'éventuelles difficultés de compréhension, sont définies en vue de les distinguer.

Séquence de didactisation $2^{\circ} a$ : autour de la différence entre message et référent Le message c'est l'objet du texte..........(séquence enchâssée) ..........les idées c'est de l'information......... (retour à séquence $2^{\circ} \mathrm{a}$ ) le message c'est l'ensemble des informations qu'on va transmettre......... (retour à la séquence enchâssée) ..........les idées sont incluses dans les informations..........dans une lettre par exemple, la formule de politesse, contrairement aux autres énoncés du texte, n'est pas une idée mais une information......... (retour à la séquence $2^{\circ} \mathrm{a}$ ) le référent c'est ce à quoi nous renvoie d'une manière précise le message.

La définition du référent retient particulièrement notre attention. Elle est effectuée en s'appuyant sur celle de message. On peut reconnaître ici un cheminement courant en 
enseignement, aller du connu vers le nouveau. Par ailleurs, dans cette même séquence, une autre, enchâssée à deux reprises, est identifiée. Son but est d'amener à établir une distinction entre "idée» et «information», laquelle rentre dans la définition du message.

Dans la séquence $2^{\circ} \mathrm{b}$ ( $\mathrm{Cf}$. infra) portant sur l'interprétabilité du message, l'idée qu'il existerait "quelque chose» qui échapperait au lecteur dans la lecture d'un écrit, évoquée dans la séquence $1^{\circ}$, trouve un élargissement explicatif à travers l'intégration de la notion d'intention de communication.

Séquence de didactisation $2^{\circ} \mathbf{b}:$ autour de l' « interprétabilité » du message

Le message peut être compris différemment d'un lecteur à un autre.........il pourrait avoir de ce fait plusieurs interprétations (référents) ..........donc, on ne peut pas chercher le sens d'un texte [...] parce que cela dépend de l'intention [...] et l'intention ne peut être connue du lecteur car celui-ci n'est pas présent dans la situation de production du texte.

Ce «quelque chose» est à ramener à la non-connaissance de l'intention de communication du scripteur, que seul ce dernier détient au sens propre du terme, et ce, en raison de la non-présence du lecteur dans la situation d'énonciation. En l'absence de l'intention en question et de tout autre élément relevant de l'univers de la production, on aura affaire à un "sens ouvert » tel que l'appelle P. Charaudeau (1994) :

J'aurais envie de dire pour ma part qu'un texte considéré hors de ses circonstances de production est en effet porteur de sens, mais d'un sens ouvert, pluriel, non encore domestiqué, témoignant de multiples voix (le "ça" de R. Barthes).

14 La progression des savoirs co-construits par les acteurs de la situation pédagogique se poursuit dans le sens de la définition du texte. En fait, il ne s'agit là que du prolongement de ce qui est abordé jusqu'à maintenant car, sous une forme ou une autre, tout s'inscrit dans une définition large de l'écrit. Dans un noyau de didactisation indépendant (Cf. infra. Extrait A), l'enseignant valide la réponse d'un apprenant qui définit le texte comme un « moyen de communication ».

Extrait $\mathrm{A}$ : noyau de didactisation indépendant $\mathrm{A}^{4}$ : la notion de texte

$\mathrm{E}:$ Ah ! ça y est, ça y est, ça y est, on passe, ça y est, c'est dit, c'est juste, un ensemble d'informations euh, on va un petit peu plus loin maintenant. Qu'est-ce qu'un texte?

$\mathrm{A}:$ C'est un message.

A : C'est un moyen de communication.

E : C'est un moyen de communication, voilà ! Bain oui, puisqu'il y a des choses qui sont dites, hein?, qui sont dites par une personne qui, elle, l'a écrite en 1930 ; elle est morte et elle nous l'a laissé, et nous en tant que lecteurs, on joue, on va ouvrir le livre de, de Flaubert, on va lire; donc il y a des choses qui nous ont été dites. C'est un moyen de, de communication, d'accord?

Dans la séquence 3 ( $\mathrm{Cf}$. infra), l'enseignant part d'une représentation répandue chez certains apprenants, selon laquelle un bon texte se caractérise par les critères de simplicité et de concision, dont la subjectivité est des plus contestables, pour mettre en place une autre, plus pertinente, liée à l'organisation de l'information.

Séquence de didactisation 3 : autour de la notion de «bon texte »

On ne peut pas dire qu'un bon texte est celui qui est simple et concis les notions de simple et de concis sont relatives : elles dépendent de la connaissance de chacun.........un bon texte est un texte qui a un fonctionnement visible, reconnaissable par le lecteur.........un bon texte c'est celui qui nous permet de déterminer l'intention.........un bon texte c'est celui dont les éléments sont cohérents [...] c'est-à-dire bien liés sur différents plans : grammatical, sémantique., etc. .........traiter un texte au niveau de sa cohérence c'est le traiter au niveau de l'information. 
Dans la séquence 4 (Cf. infra), l'enseignant traite du résultat de la production écrite tout en recourant au métalangage suivant : texte, discours, extrait, énoncés.

Séquence de didactisation $4:$ autour de la notion de texte comme le résultat de la production écrite

Quand on écrit, le résultat de la production est appelé un texte ou un discours ou un extrait ou des énoncés.

Ce faisant, hormis une petite indication sur le critère de longueur dans la définition du terme " extrait », la différence entre les vocables précédents n'est pas évoquée. Or, ceci pourrait conduire les apprenants à les considérer dans leur aspect général en tant que synonymes, ce qui va à l'encontre de la stratégie de l'enseignant où s'affiche souvent une recherche de précision conceptuelle et terminologique.

Deux noyaux de didactisation indépendants succèdent à la séquence 4 . Si le premier noyau (Cf. Extrait B) s'inscrit dans la continuité du deuxième noyau de la séquence 1, à savoir " pour produire, il faut se mettre à écrire ", le deuxième (Cf. Extrait $\mathrm{C}$ ) semble contredire la séquence en question et l'intérêt qu'elle accorde au rôle de la compréhension dans la production.

Extrait B : Noyau de didactisation indépendant B : l'apprentissage de l'écrit, résultat de la pratique de l'écriture

E : Allez-y monsieur, lisez, voilà, on, on est là, hein ? Hein ? Ecrivez et lisez. Donc, pour les décrire lisez-moi ce que vous me proposez. Ecrivez, prenez du brouillant et écrivez ce que vous voulez écrire, hein ? On apprend à écrire en écrivant. Il faut toujours ramener du brouillon avec vous parce que je vais vous faire écrire de temps en temps, hein? Vous avez passé deux années du module écrit sans écrire, vous n'allez pas passer la troisième année avec moi sans écrire. Alors, allez-y, faitesle oralement.

Construite sur le modèle du célèbre proverbe "C'est en forgeant qu'on devient forgeron ", une autre représentation, la seule importante aux yeux de l'enseignant, vient ébranler la première, en mettant l'accent sur l'indispensable pratique de l'écriture avec ses tâtonnements, qui suppose trois étapes : l'écriture, la lecture et la réécriture. Le texte n'est pas le produit d'un seul jet mais une construction résultant de multiples ajustements et modifications, d'où la nécessité d'utilisation du brouillon. Seule cette réalité est significative pour l'enseignant, ni les leçons ni les explications ne peuvent remplacer la pratique effective de l'écriture.

Le noyau de didactisation $C$ se présente sous la forme suivante :

Extrait $\mathrm{C}$ : Noyau de didactisation indépendant $\mathrm{C}$ : un modèle de la production écrite

E : Alors, allez, on y va! Quelqu'un me lit, lisez, hein? Lire, lire, lire. Alors, je disais la dernière fois à ceux qui étaient là, pour arriver à écrire, il y a ce script : écrire, lire, réécrire, voilà, voilà le euh, à part ça le reste c'est la foutaise, voilà y compris ce qu'on est en train de faire.

Bien que ce noyau ait pour avantage d'orienter vers l'aspect concret de l'acte d'écrire, pensé ici en termes de "quelque chose à faire » (C. Oriol-Boyer, 1980 : 95), il n'en demeure pas moins qu'il est fortement critiqué. En effet, il est une version incomplète du modèle de la production écrite de Rohmer (cité par Cornaire et Mary Raymond, $2006: 26$ ) luimême remis en cause. Les didacticiens reprochent surtout à ce type de conception de l'acte d'écrire son ordre linéaire, en ce sens qu'il ne permet pas une dynamique de retour en arrière, une interaction entre les différentes étapes de la production mobilisées par le scripteur: pré-écriture, écriture et réécriture ${ }^{5}$ (Cornaire et Mary Raymond, Ibidem). 
ailleurs, nous ne pouvons, en examinant le noyau $C$, ne pas relever une contradiction apparente entre les dires de l'enseignant sous-tendus par une représentation " pratique » de l'acte d'écrire et ses faires successifs observés en classe lors de la réalisation des séances d'enseignement. N'est-ce pas en effet un reniement implicite des propos antérieurs dans leur quasi-totalité, que d'avancer qu'en dehors du modèle « écrire-lire-réécrire » tout est inutile ? Nous pensons qu'il y a ici une sorte de « dédoublement identitaire du je». L'un de ces je renvoie à l'enseignant en tant que représentant de l'institution sociale éducative, représentant de toute une série d'enjeux politiques et culturels, dont la mission consiste à veiller sur la formation du futur citoyen, en lui assurant une bonne transmission des connaissances, donc à travers des leçons suivant un rituel bien conventionnel. L'autre je se rattache à l'enseignantindividu auquel la longue expérience d'enseignement a appris que seule la pratique régulière d'écriture, de lecture et de réécriture offre une voie d'apprentissage réussie.

Dans les séquences qui vont suivre, la progression des savoirs enseignés est menée dans un sens bien déterminé, celui des métarègles de la cohérence textuelle de $\mathrm{M}$. Charolles (1978). Dans les propos de l'enseignant, nous identifions une inscription explicite dans cette théorie et sa terminologie : "Oui, mais je vous donne, je vous donne les, euh les, les, la terminologie qu'emploie Charolles, voilà. ». Les séquences et les noyaux de didactisation identifiés à cette étape de notre analyse sont visiblement dérivés de la séquence 3 dans la mesure où ils développent un aspect supposé par les deux derniers noyaux de la séquence en question, à savoir la cohérence textuelle.

Dans le noyau de didactisation indépendant $3^{\circ} \mathrm{a}$ ( $\mathrm{Cf}$. infra), la notion de progression est abordée.

Extrait $3^{\circ} \mathbf{a}$ : Noyau de didactisation $3^{\circ} \mathbf{a}$ : la notion de progression

$\mathrm{E}$ : Progression. Donc, le premier critère que l'on vient de voir, ..... le premier critère d'un texte cohérent, c'est un texte qui doit au moins obéir à une règle de progression, hein? Un texte doit obéir à une règle de progression ; c'est-à-dire un texte doit progresser, progresser, progresser dans l'information.

La séquence suivante, $3^{\circ} \mathrm{b}$ ( $\mathrm{Cf}$. infra.) porte de manière anticipée sur la métarègle de répétition (la continuité thématique). Le premier noyau de didactisation indique qu'il est inutile de répéter littéralement le thème dans une deuxième phrase. Le deuxième noyau quant à lui traite du rapport entre le changement du sujet et la ponctuation, phénomène également d'ordre textuel, bien que le terme "sujet», employé par l'enseignant, nous renvoie davantage à la syntaxe de la phrase.

Séquence de didactisation $3^{\circ} \mathrm{b}$ : autour de la notion de répétition : la forme de répétition du thème

Il est inutile de répéter (identiquement) le sujet (le thème) dans une deuxième phrase ..........quand on commence un nouveau sujet, on ne met pas de virgule......... mais un point.

Le deuxième noyau de cette séquence $\left(3^{\circ} \mathrm{b}\right)$ est d'emblée discutable car il est nettement réducteur dans la mesure où il s'oppose à ce qui est affirmé dans certaines recherches en linguistique et en psycholinguistique ${ }^{6}$, concernant la complexité des usages de la ponctuation. En effet, les signes de ponctuation peuvent remplir plusieurs fonctions : sémantique, énonciative, intonative ; et de ce fait, aborder un nouveau sujet n'implique pas nécessairement de mettre un point avant, comme le présente l'enseignant, et l'intervention de tous les niveaux de considération est ce qui détermine la possibilité ou non de cette opération. On ne peut ignorer aussi le rôle des particularités stylistiques des scripteurs dans un tel choix (F. Rink, 2007 : 123-124). 
L'autre séquence que nous distinguons est celle désignée par $3^{\circ}$ aa (cf. infra). Nous précisons d'abord que cette séquence est non seulement dérivée de la séquence 3, mais aussi du noyau de didactisation $3^{\circ} \mathrm{a}$, qu'elle développe.

Séquence de didactisation $3^{\circ} \mathbf{a a}$ : retour à la notion de progression

Un texte est acceptable s'il présente des informations différentes..........un texte n'est pas acceptable s'il tourne autour du pot.........un texte peut être acceptable linguistiquement (grammaire, vocabulaire, orthographe) mais ne l'est pas textuellement..........pour qu'un texte soit cohérent, il ne suffit pas qu'il ait des informations nouvelles..........mais il faut que celles-ci soient reliées entre elles..........par un thème..........sinon le texte progressera mal.

La séquence $3^{\circ}$ aa constitue donc un prolongement du noyau de didactisation indépendant $3^{\circ} \mathrm{a}$, présenté plus haut, qui traite de la métarègle de progression. Cette dernière est considérée ici comme caractéristique inhérente au texte acceptable par opposition au texte qui ne l'est pas parce que répétitif. Dans un deuxième noyau, il est fait une distinction entre l'acceptabilité textuelle et l'acceptabilité grammaticale en ce sens qu'un texte, bien que parfaitement grammatical, pourrait ne pas être correct sur le plan textuel (cohérence). Dans cette même séquence, la notion de progression est considérée dans ses limites : en évoquant de nouvelles informations, on doit tisser des liens avec celles qui précèdent et avec le thème général du texte.

\section{Les séquences et les noyaux de didactisation dans la séance 2}

Dans la deuxième séance d'enseignement analysée, nous identifions 4 séquences de didactisation. Elles sont toutes dérivées d'une autre appartenant à la séance antérieure, la séquence 3, dont elles développent chacune un aspect livré implicitement, à savoir la cohérence textuelle. Ces séquences traitent respectivement les notions suivantes: la répétition ou la continuité thématique ( $\mathrm{Cf}$. Séquence $3^{\circ} \mathrm{bb}$ ), la non-contradiction (Cf. Séquence $3^{\circ} \mathrm{C}$ ), la relation (Cf. Séquence $3^{\circ} \mathrm{d}$ ) et enfin la nature textuelle ou extratextuelle des métarègles de la cohérence textuelle ( $\mathrm{Cf}$. Séquence $3^{\circ} \mathrm{dd}$ ). Notons que les séquences, $3^{\circ} \mathrm{bb}$ et $3^{\circ} \mathrm{dd}$, sont doublement dérivées : d'une part de la séquence 3 , et de l'autre, de séquences dont elles constituent le prolongement: il s'agit respectivement des séquences $3^{\circ} \mathrm{b}$, figurant dans la séance 1 , et $3^{\circ} \mathrm{d}$ relevant de la présente séance.

S'agissant des séquences enchâssées, la première apparaissant dans la séquence $1^{\circ}$ traite de la définition du texte, et la deuxième figurant dans la séquence $2^{\circ} \mathrm{a}$, a pour objet l'« idée ».

Séquence de didactisation $3^{\circ} \mathrm{bb}$ : retour à la notion de répétition

Un texte n'est pas acceptable si ses phrases ne sont pas enchainées..........si chaque

phrase présente son propre thème......... Un texte, pour être cohérent, doit

répéter le thème..........cependant, il ne s'agit pas de répéter le thème

littéralement.........mais de le faire substituer.........par des substituts grammaticaux..........ou lexicaux.

La séquence de didactisation $3^{\circ} \mathrm{bb}$ est articulée autour de la métarègle de répétition (la continuité thématique), déjà abordée dans l'un de ses aspects, l'inutilité d'une reprise littérale du thème dans une nouvelle phrase, dans la séquence $3^{\circ} \mathrm{b}$. Elle n'est cependant à percevoir comme dérivée de cette dernière que partiellement, car outre les éléments permettant d'éviter la répétition dans un texte, à savoir les substituts grammaticaux et lexicaux, d'autres, liés à la définition même de la répétition sont donnés. 
Les séquences $3^{\circ} \mathrm{C}$ et $3^{\circ} \mathrm{d}$ (Cf. infra) traitent respectivement des notions de noncontradiction et de relation.

Séquence de didactisation $3^{\circ} \mathrm{C}$ : autour de la notion de non- contradiction Un texte acceptable au niveau de sa progression et de sa continuité, peut ne pas être acceptable à un autre niveau parce qu'il comporte une contradiction.......... donc, pour qu'il ait un texte cohérent, il ne faut pas se contredire..........la contradiction peut résulter d'un oubli, ce qui crée une absence de continuité. Séquence de didactisation $3^{\circ} \mathrm{d}$ : autour de la notion de relation Un texte n'est pas acceptable s'il présente une contradiction avec la réalité..........la réalité sociale, la réalité psychologique, la réalité culturelle..........d'un lecteur donné......... donc, il y a deux types de contradictions: l'une extratextuelle (relation), l'autre textuelle (non-contradiction)

Ici encore, comme pour la séquence $2^{\circ}$ a (cf. supra), les savoirs nouvellement introduits reposent sur d'autres ayant déjà fait l'objet d'une didactisation. Ainsi, la métarègle de relation, objet principal de la séquence $3^{\circ} \mathrm{d}$, est définie par rapport à celle de noncontradiction, abordée dans la séquence $3^{\circ} \mathrm{C}$. Un texte qui n'est pas cohérent du point de vue de la relation est bien un texte qui présente une contradiction, mais cette fois-ci à un niveau extratextuel, par rapport au monde du réel.

Par ailleurs, il y a nécessité de nuancer la conception de la relation présentée dans la séquence $3^{\circ} \mathrm{d}$, en distinguant entre la logique du réel et celle de la fiction, ce qui n'est pas fait par l'enseignant. En effet, l'important n'est pas de savoir si un écrit est conforme à la réalité, mais de le situer dans l'un des mondes envisageables qu'il soit réel ou imaginaire. Ainsi, un conte de fées, bien que s'inscrivant dans l'irréel, ne serait pas moins cohérent sur le plan de la relation qu'un article de vulgarisation scientifique, et ce, dès que les opérations d'encodage et celles de décodage se placent dans la logique de ce genre. C. Vandendorpe (1995 : 19) explique ce détail important comme suit :

Le jugement de cohérence ou d'incohérence est donc, dans une certaine mesure, relatif à une pratique textuelle déterminée. Le texte absurde à la Ionesco ou le monologue humoristique à

la Yvon Deschamps ne sont pas régis par les mêmes lois que le texte informatif: ils en constituent même des antithèses assez exactes, sur plusieurs aspects.

Dans la séquence $3^{\circ} \mathrm{C}$, un élément nous interpelle. Pour la première fois, un défaut de cohérence est ramené à une raison l'expliquant, ici l'oubli. Cette raison semble la plus évidente car il y a peu de chance de se retrouver avec un problème de maîtrise de la langue en parlant de la cohérence à ce niveau. Un scripteur s'imaginerait mal, sauf dans des cas stylistiques particuliers, dans cette situation paradoxale d'affirmation et d'infirmation de la même chose. Même avec un bagage linguistique restreint, on estime en effet qu'un scripteur est capable d'éviter ce genre d'anomalies. Par ailleurs, il nous semble qu'il serait plus adéquat de recourir également à l'explication des autres défauts de cohérence et surtout d'orienter vers les solutions les plus efficaces afin de les éviter.

21 La séquence $3^{\circ} \mathrm{dd}$ (Cf. infra.), la dernière, s'inscrit dans une certaine mesure, dans le prolongement du dernier noyau de la séquence $3^{\circ} \mathrm{d}$.

Séquence de didactisation $3^{\circ} \mathrm{dd}$ : retour aux notions de règle textuelle et de règle extratextuelle

La règle de continuité est une règle d'ordre textuel ......... la règle de progression est aussi textuelle..........la règle de non- contradiction est également textuelle.

En effet, la nature textuelle ou non des métarègles de contradiction et de relation est reprise dans la séquence $3^{\circ} \mathrm{dd}$ pour rendre compte du reste des métarègles de la cohérence à savoir la répétition et la progression. Cette transposition d'un aspect concernant initialement une partie d'un ensemble est susceptible de donner à l'apprenant une représentation plus complète de la cohérence textuelle. 
L'analyse des objets enseignés dans le corpus recueilli nous a permis de relever plusieurs insuffisances concernant leur choix et leur progression. Du côté de leur choix, certains d'entre eux sont largement dépassés aussi bien en didactique que dans les disciplines de référence, c'est le cas de la notion de typologie textuelle et du modèle de la communication de R. Jakobson. D'autres objets sont réduits à certaines réalités, ce qui va à l'encontre de leur complexité au niveau des emplois. C'est ce que nous avons observé dans l'explication de la règle de non- contradiction où il $\mathrm{y}$ a une confusion entre les logiques du réel et de la fiction. Nous retrouvons le même phénomène dans la séquence consacrée à la notion de compréhension où il est fait abstraction totale du rôle des connaissances antérieures du sujet, et dans l'explication de la fonction du point qui ne tient pas compte des diverses considérations liées à l'usage des signes de ponctuation, notamment celles en rapport avec le style du scripteur. Du côté de la progression des savoirs enseignés, nous remarquons une certaine souplesse dans la mesure où les retours en arrière à travers les séquences de didactisation dérivées sont fréquents. La présence de séquences enchâssées dans d'autres à des moments bien déterminés renforce également cette souplesse.

\section{BIBLIOGRAPHIE}

ADAM J.-M., « La notion de typologie de textes en didactique du français. Une notion « dépassée »? ", in Recherches, $n^{\circ}$ 42, 2005, disponible sur : [http://www.unil.ch/files/live//sites/ fra/files/shared/Typologie_de_textes_et_didactique.pdf]

AMMOUDEN M., L'apprentissage actif de l'écrit et/ou de l'oral en licence de français dans le cadre d'une approche intégrée, Thèse de doctorat en didactique des langues et des textes, Université de Bejaia, EDAF (Dirs. M. Kebbas \& C. Cortier), 2012. BRASQUET-LOUBEYRE M., « Marques de didacticité dans des discours de vulgarisation scientifique à la radio ", in Les Carnets du Cediscor, $\mathrm{n}^{\circ}$ 2, 1994, disponible sur https://journals.openedition.org/cediscor/585.

CARTIER S. et VIAU R., « Application à l'élève en difficulté d'apprentissage de quatorze principes pour une réforme scolaire centrée sur l'apprenante et l'apprenant ", Site de l'adaptation scolaire et sociale de langue française, 1999, pp. 1-10, disponible sur [http://www.adaptationscolaire.net/ themes/diap/documents/lectures_diap_application.pdf].

CHARAUDEAU P., « Les conditions de compréhension du sens du discours », Revue ICI et LÀ, Madrid, Soc. General Española de Librería, 1994, disponible sur [http://www.patrickcharaudeau.com/Les-conditions-de-comprehension-du,62.html]

CHAROLLES M., «Introduction aux problèmes de la cohérence des textes », in Charolles \& Paytard J. (dir.), Langue française, $\mathrm{n}^{\circ} 38,1978$, Larousse, pp. 7-41, disponible sur [http:// www.persee.fr/articleAsPDF/lfr_0023-8368_1978_num_38_1_6117/ article_lfr_0023-8368_1978_num_38_1_6117.pdf].

CHARTRAND S.-G., et al, Progression dans l'enseignement du français langue première. Répartition des genres textuels, des notions, des stratégies et des procédures à enseigner de la 1re à la 5e secondaire, Québec, Les Publications Québec français, Numéro hors-série, 2008, (consulté en septembre 2012). 
CHEVALLARD Y., « Pourquoi La Transposition Didactique ? », Communication au Séminaire de didactique et de pédagogie des mathématiques de LIMAG, Grenoble, Université scientifique et médicale, 1982, pp. 167-194, disponible sur [http://yves.chevallard.free.fr/spip/spip/IMG/pdf/ Pourquoi_la_transposition_didactique.pdf]

CONSEIL DE L'EUROPE, Un cadre européen commun de référence pour les langues : apprendre, enseigner, évaluer, Strasbourg, Division des politiques linguistiques, 2001, disponible sur [http:// www.coe.int/t/dg4/linguistic/Source/Framework_FR.pdf] CORNAIRE C. \& Mary RAYMOND P., La production écrite, Liège, CLE International, 2006.

CUQ J.-P. GRUCA I., Cours de didactique du français langue étrangère et seconde, Grenoble, PUG, 2003.

GARCIA-DEBANC C., «Une méthodologie pour déterminer les objets effectivement enseignés : l'étude des reformulations dans l'interaction didactique. Étude de cas d'une séance conduite par un enseignant débutant en fin d'école primaire ", in Schneuwley B \& Thevenaz-Christen T. (dir.), Analyse des objets enseignés. Le cas du français, Bruxelles, De Boeck \& Larcier S.A., 2006, pp. 111- 141.

GERMAIN C., Évolution de l'enseignement des langues : 5000 ans d'histoire, Paris, CLE International, 1993.

HALTE J.-F., « Interaction : une problématique à la frontière », in Chiss J.-L., David J., Reuter Y. (dir.), Didactique du français. Fondement d'une discipline, Bruxelles, De Boeck \& Larcier, 2005, coll. Savoirs en pratique, pp. 61-75.

JAKOBSON R., Essais de linguistique générale, trad. Paris, Minuit, 1963.

MERCIER A., « La transposition des objets d'enseignement et la définition de l'espace didactique, en mathématiques ", in Revue Française de Pédagogie, n 141, 2002, Institut national de recherche pédagogique, pp. 135-171, disponible sur http://www.persee.fr/web/revues/home/prescript/ article/rfp_05567807_2002_num_141_1_2922]

MOIRAND S., Situations d'écrit, Compréhension, production en langue étrangère, Paris, Nathan/CLE international, 1979.

ORIOL-BOYER C., « Lire et écrire. Atelier d'écriture et formation des maitres ", in Pratiques, $\mathrm{n}^{\circ} 26$, 1980, pp. 94-112, disponible sur [http://www2.ac-lyon.fr/centres/delay/IMG/

pdf_Lire_pour_ecrire_Oriol-Boyer_reduit.pdf]

PASSERAULT J.-M., « La ponctuation : recherches en Psychologie du Langage », in Bessonnat D. (dir.), Pratiques, n 70, 1991, pp. 85-103, disponible sur [http://www.pratiques-cresef.com/ p070_pa1.pdf]

PUREN C., Histoire des méthodologies de l'enseignement des langues, Paris, Nathan-CLE international, 1988, coll. Didactique des Langues Étrangères, disponible sur [http://www.christianpuren.com/ mes-travaux/1988a2/]

RINCK F., «Styles d'auteur et singularité des textes. Approche stylométrique du genre de l'article en linguistique », in Petitjean A \& Rabatel A., Pratiques, n 135-136, 2007, pp. 119-136, disponible sur [http://www.pratiques-cresef.fr/p135_ri1.pdf]

VANDENDORPE Christian, « Au-delà de la phrase : la grammaire du texte », in Chartrand S., Pour un nouvel enseignement de la grammaire, Montréal, Éditions Logiques, 1995, disponible sur [http:// www.lettres.uottawa.ca/vanden/Grammaire\%20du\%20texte.pdf].

WAENDENDRIES M., « Le guidage du dialogue en classe de langue », in Cicurel F et Blondel E. (dir.), Les Carnets du Cediscor, $\mathrm{n}^{\circ} 4,1996$, disponible sur [https://journals.openedition.org/ cediscor/429]. 
WAMBACH CIAVER M., « Méthodologie des langues - Pédagogie différenciée - Réflexions pédagogiques et propositions de matériaux pour le travail en UF7 - Unité de préparation des apprenants à l'épreuve intégrée ", Le Point sur la Recherche en Education, $n^{\circ} 21,2001$, pp. 13-42, disponible sur [http://www.enseignement.be/download.php?do_id=1902\&do_check =]

\section{NOTES}

1. Selon M. Brasquet-Loubeyre (1994), le concept de didacticité est attribué à J-C. Beacco (1980), A. Bouacha (1984), Jacobi (1984). Il réfère à plusieurs dimensions caractéristiques du discours didactique : progressivité, explication, mise en relief, etc.

2. Nous introduisons chaque séquence de didactisation par un titre correspondant à l'objet principal autour duquel elle est articulée. Les éléments de la séquence, séparés par des pointillés, constituent les noyaux de didactisation qui, en vue de garder un certain aspect d'authenticité, reprennent, pour la plupart, les propos mêmes de l'enseignant de notre corpus. Cependant, nous avons décidé de nous limiter dans cette tâche aux éléments essentiels. L'emploi des crochets indique la suppression des mots ou expressions que nous jugeons superflus (généralement des reformulations).

3. Les apprenants, dans le contexte qui nous intéresse, disposent d'une certaine expérience en matière de réception et de production textuelle, aussi bien en langue étrangère, le français, qu'en langue maternelle ou de scolarisation, à savoir respectivement le berbère et l'arabe.

4. Pour une meilleure lisibilité, nous avons préféré insérer les noyaux de didactisation indépendants dans leurs contextes tout en les mettant en gras.

5. La réécriture dans le modèle de Rohmer n'est susceptible d'apporter que des corrections superficielles à la rédaction.

6. Pour une synthèse de ces recherches, voir J-M. Passerault (1991).

\section{RÉSUMÉS}

En classe de langue, le contenu notionnel, dans sa variété épistémologique, se trouve au cœur de la pratique enseignante et ses incidences sur l'apprentissage ne sont pas sujettes à contestation. L'analyser est de ce fait une nécessité d'un point de vue didactique. La présente contribution s'inscrit dans cette perspective de recherche qui se veut une concrétisation d'un principe constituant l'un des fondements de la science d'enseignement, à savoir l'étude de la transposition didactique (Y. Chevallard, 1982). Notre analyse porte sur un corpus de séances pédagogiques de FLE, filmées et transcrites. Nous avons pour objectif, d'une part, de mettre en évidence les savoirs enseignés ainsi que de les évaluer à la lumière des données théoriques actuelles en didactique des langues ; d'autre part, d'envisager ces savoirs dans leur organisation et leur progression en nous appuyant sur les notions de noyau et de séquence de didactisation.

In language classes, the notional content, in its epistemological dimension, is found at the heart of the teaching practice and its incidences on learning is not subject to contestation. Its analysis is therefore, a need from a didactic point of view. The present contribution stands to realize the ideal which constitutes one of the principles of the teaching foundations in didactics as a discipline, namely the study of didactic transposition (Y. Chevallard, 1982).Our analysis is based 
on a corpus of educational sessions of FFL (French as a Foreign Language),filmed and transcribed. Our goal is, on the one hand, to highlight the knowledge taught and to evaluate it in the light of the current theoretical data in language teaching; and on the other hand, to consider the organization and progress of this knowledge relying on the concepts of 'core' and 'sequence' of 'didactisation'.

INDEX

Mots-clés : savoirs enseignés, noyau de didactisation, séquence de didactisation, transposition didactique, apprentissage

Keywords : taught knowledge, core of didactisation, sequence of didactisation, didactics transposition, learning

\section{AUTEURS}

\section{KHEDOUDJA MEZIANE}

Doctorante - Laboratoire de recherche formation LAILEMM Université de Bejaia

\section{ABDENOUR AREZKI}

Directeur de recherche - Laboratoire de recherche formation LAILEMM Université de Bejaia 